\title{
The third electromagnetic constant of an isotropic medium
}

\author{
José F. Nieves \\ Department of Physics, University of Puerto Rico, Rio Piedras, PR 00931, USA \\ Palash B. Pal \\ Center for Particle Theory, University of Texas, Austin, TX 78712, USA.
}

\begin{abstract}
In addition to the dielectric and magnetic permeability constants, another constant is generally needed to describe the electrodynamic properties of a linear isotropic medium. We discuss why the need for the third constant arises and what sort of physical situations can give rise to a non-zero value for it. This additional constant, which we call the "Activity Constant" and denote by $\zeta$, can explain optical activity and other phenomena from a purely macroscopic and phenomenological point of view.
\end{abstract}

\section{Introduction}

In principle, the best way to discuss electromagnetic processes taking place within a material medium is to use the subatomic picture in which matter is but a huge number of fundamental particles crowded in the vacuum. The charges and currents of these particles can be fed into the equations of vacuum electrodynamics. The resulting equations, if solved, can describe all electromagnetic phenomena.

In practice, however, this is not a viable approach because, as already commented, the number of charges in any macroscopic sample of a material is enormous. One, therefore, has to take a simplified approach. This is achieved by dividing all the sources of charge into two parts: the external and the induced. The external charges are described by constructing the charge- and current- densities of them and feeding them into Maxwell's equations. The presence of the induced charges is taken into account by introducing a few empirical constants in the equations.

The number of such constants necessary depends on the nature of the medium in question. For the simplest isotropic materials, it is well known that one needs two quantities, the dielectric constant $\epsilon$ and the magnetic permeability $\mu$, to describe all phenomena of macroscopic electrodynamics [1]. However, for certain isotropic systems one needs in general three constants (not two) to describe electrodynamic phenomena in them. While this fact is also well known [2], it is not widely discussed in standard physics textbooks on electromagnetism [3].

In this paper we explain, in simple terms, why the third constant (which we denote by $\zeta$ ) arises and what kind of situations might lead to the vanishing of this extra constant. Some interesting physics associated with $\zeta$ is discussed. This includes the phenomena of optical activity of some active solutions, and of atomic 
Table 1: Electromagnetic quantities and their dimensions, in Heaviside-Lorentz $(\mathrm{HL})$ units, in terms of the fundamental units of length $(L)$, time $(T)$ and electric charge $(Q)[6]$. To find the dimensions of the Fourier transforms of these quantities, multiply any entry by $L^{3} T$. In the last two columns, we give the recipe to transform the quantities in other systems of units.

\begin{tabular}{|c||l|c|c|}
\hline \multirow{2}{*}{ Quantity } & Dimension & \multicolumn{2}{|c|}{ Replace by the following to change to } \\
\cline { 3 - 4 } & in HL units & Gaussian units & SI units \\
\hline$\rho$ & $Q L^{-3}$ & $\sqrt{4 \pi} \rho$ & $\rho / \sqrt{\epsilon_{0}}$ \\
$\mathbf{j}$ & $Q L^{-2} T^{-1}$ & $\sqrt{4 \pi} \mathbf{j}$ & $\mathbf{j} / \sqrt{\epsilon_{0}}$ \\
$\mathbf{E}$ & $Q L^{-2}$ & $\mathbf{E} / \sqrt{4 \pi}$ & $\sqrt{\epsilon_{0}} \mathbf{E}$ \\
$\mathbf{B}$ & $Q L^{-2}$ & $\mathbf{B} / \sqrt{4 \pi}$ & $\mathbf{B} / \sqrt{\mu_{0}}$ \\
$c$ & $L T^{-1}$ & $c$ & $1 / \sqrt{\mu_{0} \epsilon_{0}}$ \\
\hline
\end{tabular}

parity violation which arises due to the breaking of the parity symmetry in the weak interactions. Because $\zeta$ is crucial in explaining these phenomena from a macroscopic point of view, we refer to it as the "Activity constant". A discussion of the same issues from a quantum field theoretic point of view can be found in Ref. 海 and Ref. [5].

\section{Formulation of Macroscopic Electrodynamics}

In the vacuum, Maxwell's equations consist of two homogenous equations in the electric field $\mathbf{E}$ and the magnetic field $\mathbf{B}$ :

$$
\nabla \cdot \mathbf{B}=0 \quad, \quad c \nabla \times \mathbf{E}=-\frac{\partial \mathbf{B}}{\partial t}
$$

and two inhomogeneous equations involving the sources:

$$
\nabla \cdot \mathbf{E}=\rho \quad, \quad c \nabla \times \mathbf{B}-\frac{\partial \mathbf{E}}{\partial t}=\mathbf{j} .
$$

We have used the Heaviside-Lorentz units here so that no factors of $4 \pi$ appear in the equations. On the other hand, Coulomb law and Biot-Savart law appear now with factors of $(4 \pi)^{-1}$. As usual, $c$ is a universal constant denoting the magnitude of the velocity of light in the vacuum. For rewriting any equation of the present paper in other popular systems of units, see Table 1 .

For future purposes, let us note the dimensions of various electromagnetic quantities in these units. Of course, $\rho$ has the dimension of $Q L^{-3}$ since, by definition, it is the charge per unit volume [6]. Once that is known, Eqs. (2.1) and (2.2) determine the dimensions of $\mathbf{E}, \mathbf{B}$ and $\mathbf{j}$. The results are summarized in Table 1.

It is convenient to re-write all the above equations in terms of their Fourier components. We therefore express every quantity $\Phi(\mathbf{x}, t)$ as a superposition of plane waves through the relation [7]

$$
\Phi(\mathbf{x}, t)=\frac{1}{(2 \pi)^{2}} \int d \omega \int d^{3} \mathbf{k} \widetilde{\Phi}(\mathbf{k}, \omega) \exp [i(\mathbf{k} \cdot \mathbf{x}-\omega t)] .
$$

From the usual results of Fourier analysis, it is then easy to show that

$$
\nabla \rightarrow i \mathbf{k}, \quad \partial / \partial t \rightarrow-i \omega
$$


which indicates how the derivatives on co-ordinate space functions affect the Fourier components. With the aid of these properties, we can cast Maxwell equations in the form

$$
\begin{array}{rll}
\mathbf{k} \cdot \mathbf{B}=0 & , & c \mathbf{k} \times \mathbf{E}=\omega \mathbf{B} \\
i \mathbf{k} \cdot \mathbf{E}=\rho & , & i c \mathbf{k} \times \mathbf{B}+i \omega \mathbf{E}=\mathbf{j} .
\end{array}
$$

This form makes the ensuing discussion much easier since the equations become algebraic. This is especially convenient later when we introduce the dielectric constant $\epsilon$ and the magnetic permeability $\mu$ of a medium through Eqs. (2.10) and (2.13), which would become integral relations in co-ordinate space. Notice that we write the Fourier components with the same notation as the space-time functions themselves. This will not cause any confusion because the space-time functions will never again be used in this article.

As mentioned in the Introduction, the discussion of macroscopic electrodynamics starts from the observation that $\rho$ and $\mathbf{j}$ contain, in addition to any external sources (denoted by the subscript "ext"), the sources induced in the medium (denoted by the subscript "ind"):

$$
\rho=\rho_{\text {ext }}+\rho_{\text {ind }} \quad, \quad \mathbf{j}=\mathbf{j}_{\text {ext }}+\mathbf{j}_{\text {ind }} .
$$

Ordinarily, the induced sources are expressed in terms of the polarization $(\mathbf{P})$ and the magnetization $(\mathbf{M})$ as follows:

$$
\begin{aligned}
\rho_{\text {ind }} & =-i \mathbf{k} \cdot \mathbf{P} \\
\mathbf{j}_{\text {ind }} & =-i \omega \mathbf{P}+i c \mathbf{k} \times \mathbf{M} .
\end{aligned}
$$

In this form, the expressions for $\rho_{\text {ind }}$ and $\mathbf{j}_{\text {ind }}$ mimic those for total $\rho$ and $\mathbf{j}$ given in Eq. (2.6), and they automatically satisfy the equation of continuity, which takes the following form in Fourier space:

$$
\mathbf{k} \cdot \mathbf{j}_{\mathrm{x}}-\omega \rho_{\mathrm{x}}=0
$$

where the subscript ' $\mathrm{x}$ ' stands for the induced sources. This signifies the conservation of induced charges. The conservation equation (2.9) also holds when ' $x$ ' stands for the total or just the external charges.

The dielectric constant $\epsilon$ and the magnetic permeability $\mu$ for a system are now introduced by writing

$$
\mathbf{P}=(\epsilon-1) \mathbf{E} \quad, \quad \mathbf{M}=\left(1-\mu^{-1}\right) \mathbf{B} .
$$

In writing these relations it is assumed, as we do in this paper, that the response of the system to applied electromagnetic fields is linear. This excludes special systems such as ferroelectrics and ferromagnetics from consideration, but otherwise does not impose any real restriction provided that the applied fields are not extremely large. Further, we will consider the simple case of isotropic media, for which $\epsilon$ and $\mu$ can be taken as scalars [8]. One can then use Eqs. (2.7), (2.8) and (2.10) to rewrite Eqs. (2.5) and (2.6) as

$$
\begin{array}{rll}
\mathbf{k} \cdot \mathbf{B}=0 & , \quad c \mathbf{k} \times \mathbf{E}=\omega \mathbf{B} \\
i \mathbf{k} \cdot \mathbf{D}=\rho_{\text {ext }} & , \quad i c \mathbf{k} \times \mathbf{H}+i \omega \mathbf{D}=\mathbf{j}_{\text {ext }} .
\end{array}
$$

where

$$
\mathbf{D} \equiv \mathbf{E}+\mathbf{P}=\epsilon \mathbf{E} \quad, \quad \mathbf{H}=\mathbf{B}-\mathbf{M}=\mu^{-1} \mathbf{B}
$$

Since the source terms are just the external charges and currents, one can in principle solve Eq. (2.12) for $\mathbf{D}$ and $\mathbf{H}$. Once the empirical constants $\epsilon$ and $\mu$ are known, one can then use Eq. (2.13) to derive $\mathbf{E}$ and $\mathbf{B}$.

In the next section, we will see that this treatment makes an important assumption tacitly, and because of that, it cannot describe an important class of physical phenomena within the framework of macroscopic electrodynamics. 


\section{The most general parametrization of medium properties}

\subsection{Parametrization of the induced sources}

The crucial question in macroscopic electrodynamics is: how to parametrize the induced sources $\rho_{\text {ind }}$ and $\mathbf{j}_{\text {ind }}$. It is important to recognize that these quantities cannot be determined from considerations of macroscopic physics alone. In standard textbooks of electromagnetism, some simple microscopic models of the medium are invoked to express $\mathbf{P}$ and $\mathbf{M}$ in terms of microscopic quantities, and thereby motivate the parametrization discussed above. However, such models may not describe all possible media and, therefore, the expressions in Eq. (2.8) need not be the most general ones. From a purely macroscopic point of view, we can try to parametrize $\rho_{\text {ind }}$ and $\mathbf{j}_{\text {ind }}$ in the most general form subject to the conditions of isotropy and linearity. As we show below, this approach indicates that Eq. (2.8) indeed does not provide the most general expressions for the induced charge and current densities.

To clarify the point, we summarize Eqs. (2.8) and (2.10) by writing

$$
\begin{aligned}
\rho_{\text {ind }} & =-i(\epsilon-1) \mathbf{k} \cdot \mathbf{E} \\
\mathbf{j}_{\text {ind }} & =-i(\epsilon-1) \omega \mathbf{E}+i c\left(1-\mu^{-1}\right) \mathbf{k} \times \mathbf{B} .
\end{aligned}
$$

Looked at this way, it seems that we are trying to write down $\rho_{\text {ind }}$ and $\mathbf{j}_{\text {ind }}$ as linear functions of the electric and the magnetic fields, where the coefficients depend on $\omega$ and $\mathbf{k}$. One can then ask, are the combinations the most general possible ones subject to the assumption of isotropy?

The answer is 'yes' for the expression for $\rho_{\text {ind }}$ in Eq. (3.1). Note that $\rho$ is a scalar as far as rotations are concerned, and the only scalars which are linear functions of $\mathbf{E}$ and $\mathbf{B}$ are $\mathbf{k} \cdot \mathbf{E}$ and $\mathbf{k} \cdot \mathbf{B}$. Of them, the latter is identically zero (Eq. (2.5)), so that $\rho_{\text {ind }}$ has to be proportional to $\mathbf{k} \cdot \mathbf{E}$, as shown in Eq. (3.1).

The situation is different for Eq. (3.2). Here, $\mathbf{j}_{\text {ind }}$ is a vector, and there are four vectors involving $\mathbf{E}$ and $\mathbf{B}$, viz., $\omega \mathbf{E}, c \mathbf{k} \times \mathbf{B}, \omega \mathbf{B}$ and $c \mathbf{k} \times \mathbf{E}$, which have the same dimensions as $\mathbf{j}$. The last two are equal by Eq. (2.5), so that only the first three can be taken as independent. One can therefore write the most general expression for $\mathbf{j}$ as

$$
\mathbf{j}_{\text {ind }}=-i(\epsilon-1) \omega \mathbf{E}+i c\left(1-\mu^{-1}\right) \mathbf{k} \times \mathbf{B}-i \zeta \omega \mathbf{B} .
$$

Notice that the coefficient in the first term is fixed to be the same as the coefficient in Eq. (3.1) by current conservation. Thus, in general, we have three dimensionless constants describing the electromagnetic properties of an isotropic medium: $\epsilon, \mu$ and $\zeta$.

Looking back at Eq. (3.2), it now becomes immediately obvious why it is incomplete. Since $\mathbf{j}_{\text {ind }}$ is a vector having three components, one needs, in general, three basis vectors to write it down. In Eq. (3.2), we used only two basis vectors, $\omega \mathbf{E}$ and $\mathbf{k} \times \mathbf{B}$. Therefore, Eq. (3.2) is valid only under some extra assumptions, the precise nature of which will be discussed in the next section.

One comment is in order. The last equation in Eq. (2.6) expresses the total current $\mathbf{j}$ as a combination of the two vectors $\omega \mathbf{E}$ and $\mathbf{k} \times \mathbf{B}$. If we have had no complaint about that, why do we argue about Eq. (3.2), which expresses $\mathbf{j}_{\text {ind }}$ as a linear combination of the same two vectors?

The answer to this question lies in the difference of the nature of Eqs. (2.6) and (3.2). Eq. (2.6) is a law, describing the fundamental nature of electromagnetic interactions. Eq. (3.2) is a parametrization of the induced current, which expresses our ignorance about the details of the dynamics of all processes going on in the medium or our inability to quantify them in minutest details. Such parametrization must be as general as possible subject to the conditions of the problem, and Eq. (3.3) provides this general form for $\mathbf{j}_{\text {ind }}$.

With this parametrization of the induced sources, the pair of inhomogeneous equations given in Eq. (2.6) become

$$
i \epsilon \mathbf{k} \cdot \mathbf{E}=\rho_{\mathrm{ext}}
$$




$$
i \epsilon \omega \mathbf{E}+i \frac{c}{\mu} \mathbf{k} \times \mathbf{B}+i \zeta \omega \mathbf{B}=\mathbf{j}_{\mathrm{ext}}
$$

while the sourceless equations (2.5) remain intact. These two equations plus the equations in (2.5) can be taken as the fundamental equations for the $\mathbf{E}$ and $\mathbf{B}$ fields in a medium in the presence of external sources. In the next sections we will elucidate some of the physical implications and consequences associated with the constant $\zeta$.

\subsection{Constitutive relations}

As already mentioned in the Introduction, the inadequacy of the simple constitutive relations given in Eq. (2.13) for describing a certain class of physical phenomena has been recognized for a long time [2]. In many instances this deficiency has been addressed [2, 9, 10, 11] by postulating Eqs. (2.11) and (2.12) together with a more general set of constitutive relations to replace those in Eq. (2.13):

$$
\begin{aligned}
& \mathbf{D}=\epsilon \mathbf{E}+\beta \mathbf{B}, \\
& \mathbf{H}=\gamma \mathbf{E}+\mu^{-1} \mathbf{B} .
\end{aligned}
$$

With this approach, it seems that one needs two extra constants for the medium, $\beta$ and $\gamma$, rather than the constant $\zeta$ discussed earlier. However, it is easy to see that the two constants introduced here are not independent. For this, substitute Eqs. (3.5) and (3.6) into Eq. (2.12). This yields

$$
\begin{aligned}
i \epsilon \mathbf{k} \cdot \mathbf{E} & =\rho_{\mathrm{ext}}, \\
i \frac{c}{\mu} \mathbf{k} \times \mathbf{B}+i \epsilon \omega \mathbf{E}+i(\beta+\gamma) \omega \mathbf{B} & =\mathbf{j}_{\mathrm{ext}},
\end{aligned}
$$

Thus, it is only the sum $\beta+\gamma$ that appears in the field equations, and therefore only three constants have an independent physical significance. Moreover, comparing these equations with those in Eq. (3.4), we see that $\beta+\gamma=\zeta$. Because of this redundancy, one can try to put an arbitrary constraint to fix $\beta$ and $\gamma$, e.g., one might put [12] $\beta=\gamma$.

While the approach of postulating more general constitutive relations rather than the simple ones given in Eq. (2.13) has been followed in the literature for a long time, the lesson we have learned from the above discussion is that, in general, the constitutive relations that have been used contain redundant parameters. The reason for the redundancy is easy to understand. The componets of $\mathbf{E}$ and $\mathbf{B}$ are not independent. They are constrained by Eq. (2.5). Similarly, the components of $\mathbf{D}$ and $\mathbf{H}$ also are not independent. The constitutive relations, being relations between these two sets of variables none of which are independent, can also have redundant parameters. This can also be seen from Eq. 2.12). In fact, only the equation for $\mathbf{j}_{\text {ext }}$ is independent there; the equation for $\rho_{\text {ext }}$ can be derived from it using the charge conservation equation, Eq. (2.9). Taking the general expression for jext from Eq. (3.4) and eliminating B using Faraday's law, Eq. (2.11), the equation for $j_{\text {ext }}$ in Eq. (2.12) becomes

$$
c \mathbf{k} \times \mathbf{H}+\omega \mathbf{D}=\epsilon \omega \mathbf{E}+\frac{c^{2}}{\omega \mu} \mathbf{k} \times(\mathbf{k} \times \mathbf{E})+c \zeta \mathbf{k} \times \mathbf{E}
$$

Thus, we are trying to define two vectors $\mathbf{H}$ and $\mathbf{D}$ from this single equation, in terms of just $\mathbf{E}$, which is the only independent variable here. There is no unique way to do this, and any parametrization will contain some arbitrariness. In contrast, the components of $\mathbf{j}_{\text {ind }}$ are all independent. By focusing the attention on them, we have succeeded in finding a parametrization of the induced sources that is both minimal and complete. Because of this redundancy of the parametrization in terms of the constitutive relations, it is more convenient to use the parametrization of $\mathbf{j}_{\text {ind }}$ instead, which we will do in the rest of this article.

We should comment that the restriction of the number of electromagnetic constants to three applies

only in the Fourier space, which we use in the present article. In co-ordinate space on the other hand, 
Table 2: The transformation properties of various physical quantities under the operations $\mathrm{P}, \mathrm{T}$ and $\mathrm{C}$. The quantity $\eta_{P}$, for example, is defined in Eq. (4.4), and the definitions of $\eta_{T}, \eta_{C}$ etc are similar. The last two columns also show the transformation under the combined operations $\mathrm{CP}$ and $\mathrm{CPT}$.

\begin{tabular}{|c||c|c|c|c|c|}
\hline \multicolumn{1}{|c||}{} & \multicolumn{5}{c|}{ Transformation given by } \\
\hline Quantity & $\eta_{P}$ & $\eta_{T}$ & $\eta_{C}$ & $\eta_{C P}$ & $\eta_{C P T}$ \\
\hline E & - & + & - & + & + \\
B & + & - & - & - & + \\
$\mathbf{k}$ & - & + & + & - & - \\
$\omega$ & + & - & + & + & - \\
$\rho$ & + & + & - & - & - \\
$\mathbf{j}$ & - & - & - & + & - \\
A & - & - & - & + & - \\
\hline
\end{tabular}

one may add many terms using higher and higher derivatives of the $\mathbf{E}$ and $\mathbf{B}$ fields in the definition of the induced current [13]. Translated to the Fourier space, those additional terms are taken into account by allowing the constants $\epsilon, \mu$ and $\zeta$ to depend on higher powers of $\mathbf{k}$ and $\omega$.

\section{The constant $\zeta$ and the discrete symmetries of space-time}

Why is it that the constant $\zeta$ is not introduced for the simplest isotropic materials? Alternatively, what are the conditions under which $\zeta$ can have a nonzero value? The key to the answer to these questions lies in the discrete symmetries of space and time, as we discuss in this section.

\subsection{Definition of the discrete symmetries $\mathrm{P}, \mathrm{T}, \mathrm{C}$}

For orientation, consider Newton's law for a particle of mass $m$ moving under the influence of the gravitational force exerted by another particle of mass $M$ placed at the origin. The equation of motion is

$$
m \mathbf{a}=-\frac{G M m}{r^{3}} \mathbf{r}
$$

where the right hand side denotes the gravitational force on the particle. If we perform space inversion (i.e., change $\mathbf{r}$ to $-\mathbf{r}$ ), the force changes sign, but so does $\mathbf{a}$, since it is defined to be $d^{2} \mathbf{r} / d t^{2}$. So Eq. (4.1) does not change. Similarly, if we perform a time inversion (i.e., change $t$ to $-t$ ), neither side of Eq. (4.1) changes so, again, the equation remains invariant.

For a long time, it was believed that these two discrete operations, viz. of space inversion or parity $(\mathrm{P})$ and time reversal $(\mathrm{T})$, do not change any laws of physics. If this were true, all the terms in a physically acceptable equation would transform the same way under $\mathrm{P}$ and $\mathrm{T}$, as in the example discussed above. Indeed, we can verify that such is the case with the Maxwell equations in (2.1) and (2.2) or, equivalently, with their Fourier space counterparts given in Eqs. (2.5) and (2.6). To show this, we proceed as follows.

Consider, for example, Parity. The identification given in Eq. (2.4), implies that in Fourier space the parity operation is induced by the substitution

$$
(\mathbf{k}, \omega) \stackrel{P}{\longrightarrow}(-\mathbf{k}, \omega)
$$

It is very easy to verify that, if we simultanously make the following substitutions

$$
\mathbf{E}(\mathbf{k}, \omega) \stackrel{P}{\longrightarrow}-\mathbf{E}(\mathbf{k}, \omega),
$$




$$
\begin{array}{rlc}
\mathbf{B}(\mathbf{k}, \omega) & \stackrel{P}{\longrightarrow} & \mathbf{B}(\mathbf{k}, \omega), \\
\mathbf{j}(\mathbf{k}, \omega) & \stackrel{P}{\longrightarrow} & -\mathbf{j}(\mathbf{k}, \omega), \\
\rho(\mathbf{k}, \omega) & \stackrel{P}{\longrightarrow} & \rho(\mathbf{k}, \omega),
\end{array}
$$

then the equations are not changed. If for every quantity, including $\omega$ and $\mathbf{k}$ themselves, we write the above transformation rules in the form

$$
\Phi(\mathbf{k}, \omega) \stackrel{P}{\longrightarrow} \eta_{P} \Phi(\mathbf{k}, \omega),
$$

then the multiplicative constant $\eta_{P}$ has the value given in Table 2 for the various quantities.

The same procedure is followed for the Time reversal symmetry, for which Eq. (2.4) suggests [14]

$$
(\mathbf{k}, \omega) \stackrel{T}{\longrightarrow}(\mathbf{k},-\omega) .
$$

The fact that the Maxwell equations are invariant under $\mathrm{P}$ and $\mathrm{T}$ further bolstered the idea that all the laws of physics are invariant under those operations.

It is easily seen that the Maxwell equations (Eqs. (2.1) and (2.2), and their Fourier space version given in Eqs. (2.5) and (2.6)) have another discrete symmetry since they are unaffected by the operation under which $\rho, \mathbf{j}, \mathbf{E}$ and $\mathbf{B}$ all change sign. This operation, called charge conjugation (C) because the sign of all the charges are reversed, plays an important role in quantum field theory, where it converts a particle into its associated antiparticle.

\subsection{Implications of $\mathrm{P}, \mathrm{T}, \mathrm{C}$ symmetries on $\epsilon, \mu, \zeta$}

Now that we know the transformation properties of the electromagnetic fields and currents, we can look back at Eq. (3.3) with respect to the discrete symmetries C, P and T, and some important combinations like $\mathrm{CP}$ and $\mathrm{CPT}$. To proceed we first notice that we can consider instead

$$
\mathbf{j}_{\mathrm{ext}}(\mathbf{k}, \omega)=i \epsilon(k, \omega) \omega \mathbf{E}(\mathbf{k}, \omega)+\frac{c}{\mu(k, \omega)} i \mathbf{k} \times \mathbf{B}(\mathbf{k}, \omega)+i \zeta(k, \omega) \omega \mathbf{B}(\mathbf{k}, \omega),
$$

which is equivalent to Eq. (3.3) because of the definition in Eq. (2.7). We have explicitly indicated the dependence on $\mathbf{k}$ and $\omega$ of all the quantities involved, because it is important to keep that in mind in what follows. In addition, we have explicitly used the fact that the quantities $\epsilon, \mu$ and $\zeta$, which represent properties of the medium, can depend on $\mathbf{k}$ only through its magnitude $k \equiv|\mathbf{k}|$ since we are considering an isotropic medium.

Before we proceed any further, we want to note that there is one relation that the electromagnetic constants $\epsilon, \mu$ and $\zeta$ must satisfy based on very general grounds. It is simply the requirement that the

fields $\mathbf{E}, \mathbf{B}$ and the current density $\mathbf{j}$ are, in coordinate space, real quantities. This implies that, for real values of $\omega$, the electromagnetic constants satisfy

$$
f^{*}(-\mathbf{k},-\omega)=f(\mathbf{k}, \omega)
$$

where $f$ stands for any of them. As noted earlier, the dependence on $\mathbf{k}$ can be only through its magnitude $k$ since we are restricting the discussion to isotropic materials. Thus, in our case, we can write

$$
f^{*}(k,-\omega)=f(k, \omega)
$$

which means that the real parts of these electromagnetic constants are even in $\omega$ whereas the imaginary parts are odd. We stress that this is a very general property, not tied up in any way with the discrete symmetry operations that we are going to discuss now. The discrete symmetries, if they are valid in any given situation, impose extra conditions on the electromagnetic constants. 
Consider, for example, Parity. The question we want to answer is the following: If we take each quantity that appears in Table 2, including $\mathbf{k}$ and $\omega$, and make in Eq. (4.6) the replacement indicated in Eq. (4.4), what relations must be satisified by $\epsilon, \mu$ and $\zeta$ in order for that equation to remain the same? In this fashion we easily derive the consequences of the parity symmetry on $\epsilon, \mu$ and $\zeta$, and similarly with the other discrete symmetries. We discuss them one by one.

Parity : The parity reversed form of Eq. (4.6) is

$$
-\mathbf{j}_{\mathrm{ext}}=-i \epsilon \omega \mathbf{E}-\frac{c}{\mu} i \mathbf{k} \times \mathbf{B}+i \zeta \omega \mathbf{B}
$$

or

$$
\mathbf{j}_{\mathrm{ext}}=i \epsilon \omega \mathbf{E}+\frac{c}{\mu} i \mathbf{k} \times \mathbf{B}-i \zeta \omega \mathbf{B} .
$$

Here and below, the arguments of $\epsilon, \mu$, and $\zeta$, unless otherwise given, should be assumed to be $k$ and $\omega$. Comparing Eqs. (4.6) and (4.10), we conclude that

$$
\text { Parity symmetry } \Rightarrow \zeta=0 \text {. }
$$

Charge Conjugation : The charge conjugated form of Eq. (4.6) is the same as the original one since each terms changes sign, and therefore we do not get any constraints on $\epsilon, \mu$, and $\zeta$.

CP : Obviously, this gives the same constraint as parity alone does, since charge conjugation does not affect Eq. (4.6), as stated above.

Time reversal : $\quad$ The time-reversed form of Eq. (4.6) is

$$
-\mathbf{j}_{\mathrm{ext}}=-i \epsilon \omega(k,-\omega) \mathbf{E}-\frac{c}{\mu(k,-\omega)} i \mathbf{k} \times \mathbf{B}+i \zeta \omega(k,-\omega) \mathbf{B},
$$

which gives, on comparison with Eq. (4.6), the following constraints:

$$
\text { Time reversal symmetry } \Rightarrow\left\{\begin{array}{rl}
\epsilon(k,-\omega) & =\epsilon(k, \omega) \\
\mu(k,-\omega) & =\mu(k, \omega) \\
\zeta(k,-\omega) & =-\zeta(k, \omega)
\end{array} .\right.
$$

Taken together with Eq. (4.8), this implies that $\zeta$ must be purely imaginary and an odd function of $\omega$, whereas $\epsilon$ and $\mu$ should be real and even functions of $\omega$.

CPT : In this case, we get similarly the following constraints

$$
\text { CPT symmetry } \Rightarrow\left\{\begin{aligned}
\epsilon(k,-\omega) & =\epsilon(k, \omega) \\
\mu(k,-\omega) & =\mu(k, \omega) \\
\zeta(k,-\omega) & =\zeta(k, \omega)
\end{aligned}\right.
$$

\subsection{Constraints on $\epsilon, \mu, \zeta$ in ordinary media}

From the relations deduced above, it is quite clear that the presence of the $\zeta$-term implies some properties that are asymmetric under P and CP transformations. Similarly, since T and CPT imply mutually inconsistent constraints on $\zeta$, at least one of them must be violated in order for $\zeta$ to exist. 
We said earlier that classically it was believed that all interactions conserve P, T and C. From this, one might have expected $\zeta$ to vanish. However, we know now that $\mathrm{P}, \mathrm{T}$ and $\mathrm{C}$ are all violated in fundamental interactions between particles.

Since 1957, we know that parity is violated by weak interactions. Since 1964, we know that CP is also violated by the same, although to a much lesser extent. Since $\mathbf{j}_{\text {ind }}$ arises because of complicated processes taking place within the medium, including weak interaction processes, there is no reason why the $\zeta$-term should not be present in Eq. (3.3).

If we leave the discussion at that point, it might seem that the parameter $\zeta$ can at best be very small, since the weak interaction is indeed very feeble. What could be worse, the $\zeta$-term violates $\mathrm{CP}$, and the CP-violating effects are known to be smaller than the usual weak interaction strength by about three orders of magnitude. So $\zeta$, if present, would seem to be extremely tiny. But that need not be true in general, as we argue shortly.

If we had carried the previous line of argument further, we would have concluded that the only constraint on the value of $\zeta$ is $\zeta(k, \omega)=\zeta(k,-\omega)$ which follows from CPT symmetry, since there are very strong reasons to believe that fundamental interactions between particles can never violate CPT. This again, would have been wrong for the reason discussed below.

In principle, the quantities $\epsilon, \mu, \zeta$ could be determined if we could solve the equations of motion of the particles that compose the medium. These equations include the effects of the mutual interactions between the particles as well as the effects of the local $\mathbf{E}$ and $\mathbf{B}$ fields. These are not external prescribed fields but are the fields that enter into Maxwell's equations, which must also be solved for, and which in turn contain the induced charge and current densities in the source terms. Thus, Maxwell's equations plus the equations of motion of the particles in the medium form a closed system which must be solved self-consistently. To date, no one has succeeded in solving this formidable problem and in practice one turns to a more modest approach.

In classical as well as quantum physics, the calculation of $\epsilon, \mu, \zeta$ proceeds in two steps. In the first, the equation of motion of a single particle is set up, which includes the effects of the local fields, as well as the effects of the other particles in the medium. From this, the contribution of a single particle to the induced charge and current is obtained. In the second step, this single-particle contribution is suitably averaged over the particles in the medium, thereby obtaining an expression for the induced charge and current densities, from which the values for $\epsilon, \mu, \zeta$ can be extracted.

It is now clear that the asymmetries with respect to any operation can enter into any quantity in either of these two steps. In the first step, it enters if the underlying interactions do not respect the symmetry generated by the operation, as is the case of weak interactions with respect to the symmetries of $\mathrm{P}, \mathrm{C}$ and $\mathrm{CP}$. But the asymmetries can also creep into the process of taking the average over the particles in the medium, because the medium might have a surplus of one kind of particles over others.

As a well-known example, consider the mass of the electron and its antiparticle, the positron. In vacuum, the two masses must be equal. Formally, this is ensured by the combined symmetry CPT. Inside a chunk of normal matter, however, there are many many electrons and hardly any positrons, so that the interactions of the two particles become very different. As a result, they move with different effective masses within a medium. Thus, the CPT breaking effects creep in through the asymmetry in the medium.

In the case of our present interest, considerations of CPT and CP symmetries are irrelevant for the same reason. In fact, the $\mathrm{CP}$ and $\mathrm{CPT}$ asymmetries are large since normal matter does not contain any antiparticles at all, and hence neither $\mathrm{CP}$ nor CPT stand in the way of obtaining a large $\zeta$.

Let us now remind ourselves that in order to obtain a large $\zeta$, we also need a large P-asymmetry. The P-symmetry is of course violated by weak interactions, and that can give rise to a small value of $\zeta$. To obtain a large $\zeta$, we need a large P-asymmetry in the medium.

Take a free electron gas, for example [15]. An electron in this gas can be specified by its momentum $\mathbf{p}$ 
and spin angular momentum $\mathbf{s}$. Under the action of parity, $\mathbf{p} \rightarrow-\mathbf{p}, \mathbf{s} \rightarrow \mathbf{s}$. However, in the metal, there are as many electrons going in the $+x$ direction, say, as in the $-x$ direction. So the parity operation does not affect the overall momentum distribution of the metallic electrons. In other words, this medium does not have any $\mathrm{P}$-asymmetry and so $\zeta$ must arise in this medium through parity violation in weak interaction.

Similarly, under the T operation, $\mathbf{p} \rightarrow-\mathbf{p}, \quad \mathbf{s} \rightarrow-\mathbf{s}$. But again, since the distributions of both $\mathbf{p}$ and $\mathbf{s}$ were random to start with, the overall distributions do not change by this operation, i.e., the medium is T-symmetric. Therefore, barring the small T-violating effects which are $10^{-3}$ times weaker than the P-violating effects in weak interactions, the consequences of T-symmetry should be taken seriously. As we found before in Eq. (4.13), this implies that $\zeta$ must be purely imaginary and an odd function of $\omega$.

This is quite striking. Note that $\epsilon$ and $\mu$ are both even functions of $\omega$, so that it is conceivable to think of a medium where they are constants. In this case the medium is non-dispersive. But $\zeta$ cannot have a nonzero constant term and therefore all media are dispersive when it comes to $\zeta$. Of course, we emphasize that this comment is true only to the extent that T-violation in fundamental interactions can be neglected.

When we consider more complicated media, it is possible to obtain P-asymmetry from the medium itself. A well-known example is that of helical molecules, with one helicity in excess over the other. This occurs in natural sugar solutions, for example, where in fact one helicity is absent altogether. If we consider the passage of electromagnetic waves through such a solution in which the helical axes are randomly oriented, and if the wavelength is larger than the molecular dimension so that the wave cannot "see" individual molecules, we have an isotropic medium with a built-in P-asymmetry. This gives rise to large $\zeta$-term, and physically it manifests itself as the phenomenon of optical activity, as we discuss in Sec. 6 .

In short, there is no reason why $\zeta$ should be zero in normal matter, and its value can be large if there exist large asymmetries in the medium. Therefore it is imperative that we understand its physical significance.

\section{Dispersion relations}

It is well-known that a great deal about the nature of wave-propagation is learned from the dispersion relations. These are the relations that $\omega$ and $\mathbf{k}$ must satisfy in order that solutions to Eq. (3.4) exist when $\rho_{\text {ext }}=0$ and $\mathbf{j}_{\text {ext }}=0$; i.e.,

$$
\begin{gathered}
\epsilon \mathbf{k} \cdot \mathbf{E}=0 \\
\epsilon \omega \mathbf{E}+\frac{c}{\mu} \mathbf{k} \times \mathbf{B}+\zeta \omega \mathbf{B}=0 .
\end{gathered}
$$

These two, plus the two equations in Eq. (2.5), can be taken as the fundamental equations governing the $\mathbf{E}$ and $\mathbf{B}$ fields in a medium in the absence of external charges and currents.

In the vacuum, since $\epsilon=\mu=1$ and $\zeta=0$, Eq. (5.1) clearly shows that $\mathbf{E}$ must be perpendicular to the direction of propagation of the electromagnetic wave. Thus, one can discuss electromagnetic waves in terms of two basis polarization vectors, $\hat{e}_{1}$ and $\hat{e}_{2}$, both being perpendicular to $\mathbf{k}$, as shown in Fig. 1.

In the medium, Eqs. (5.1) and (5.2) can be satisfied for a longitudinal electric field if $\mathbf{B}=0$ and $\omega$ is such that $\epsilon=0$. Since the physical properties of this longitudinal mode depend on the expression for $\epsilon$ only, we focus our attention on the transverse modes in order to discuss the physical implications of $\zeta$. Thus, eliminating B from Eq. (5.2) by the use of Faraday's law and using $\mathbf{k} \cdot \mathbf{E}=0$, we obtain

$$
\omega^{2} \mathbf{E}=\frac{c^{2}}{\epsilon \mu} k^{2} \mathbf{E}-\frac{c \zeta}{\epsilon} \omega k \hat{k} \times \mathbf{E},
$$

where $\hat{k}=\mathbf{k} / k$. Let us first see what happens if $\zeta=0$, which is the case treated in standard textbooks 


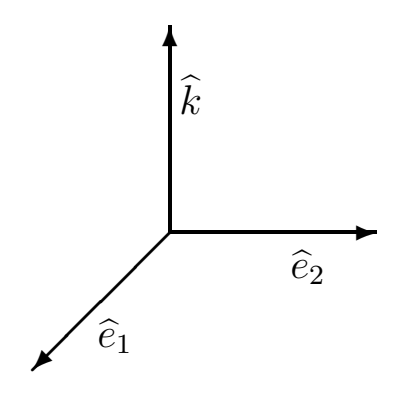

Figure 1

Figure 1: The relative orientations of the two transverse polarization vectors with respect to the wave vector.

[16]. In this case, Eq. (5.3) immediately gives the dispersion relation

$$
\omega^{2}=\frac{c^{2}}{\epsilon \mu} k^{2}
$$

In non-dispersive media (i.e. when $\epsilon$ and $\mu$ are independent of $\omega$ and $\mathbf{k}$ ), this gives the speed of propagation as $c / \sqrt{\epsilon \mu}$. Apart from the transversality condition $\mathbf{k} \cdot \mathbf{E}=0$, there is no restriction on $\mathbf{E}$. Therefore, we conclude that the two transverse modes have the same physical characteristics and, in fact, any linear combination of them is equally appropriate for discussing the transverse wave properties.

In general, however, the two transverse modes have different properties when $\zeta$ does not vanish. To proceed in this case, we take the cross product of Eq. (5.3) with $\hat{k}$ and use the transversality condition $\hat{k} \cdot \mathbf{E}=0$ to obtain

$$
\omega^{2} \hat{k} \times \mathbf{E}=\frac{c^{2}}{\epsilon \mu} k^{2} \hat{k} \times \mathbf{E}+\frac{c \zeta}{\epsilon} \omega k \mathbf{E}
$$

Taking linear combinations, this pair of coupled equations for $\mathbf{E}$ and $\hat{k} \times \mathbf{E}$ can be rewritten as

$$
\left[\omega^{2}-\left(\frac{1}{\epsilon \mu} \pm \frac{i \zeta \omega}{\epsilon k c}\right) c^{2} k^{2}\right](\mathbf{E} \pm i \hat{k} \times \mathbf{E})=0 .
$$

Therefore, solutions exist only if $\omega$ and $\mathbf{E}$ satisfy

$$
\begin{aligned}
\omega^{2} & =\left(\frac{1}{\epsilon \mu}+\lambda \frac{i \zeta \omega}{\epsilon k c}\right) c^{2} k^{2} \\
\mathbf{E} & =i \lambda \hat{k} \times \mathbf{E}
\end{aligned}
$$

with $\lambda= \pm 1$. Thus, the two transverse modes no longer have the same dispersion relation. Furthermore, an arbitrary linear combination of them does not, in general, obey the wave equation (5.3). The combinations that travel as waves are the ones that satisfy Eq. (5.8), which are in fact the positive and negative circular polarization vectors $\mathbf{e} \pm i \hat{k} \times \mathbf{e}$, where $\mathbf{e}$ is any vector transverse to $\hat{k}$ [17].

We argued before, in connection with Eq. (4.13), that $\epsilon$ and $\mu$ are real whereas $\zeta$ is purely imaginary if time-reversal invariance is satisfied. In this case, it follows from Eq. (5.7) that that $\omega$ is real for real values of $k$ if $|\zeta|<k c|\mu| / \omega$. The amplitude of a monochromatic wave will thus remain unchanged during the propagation, which means that there is no absorption in the medium. However, if $\zeta \neq 0$, the speeds of propagation of the two circlularly polarized transverse modes are different.

In a more general case, time-reversal symmetry can be violated. Then, for a given $\mathbf{k}, \omega$ is complex in general, so that the exponential factors in Eq. (2.3) give some damping (or growth) with time. Physically, 


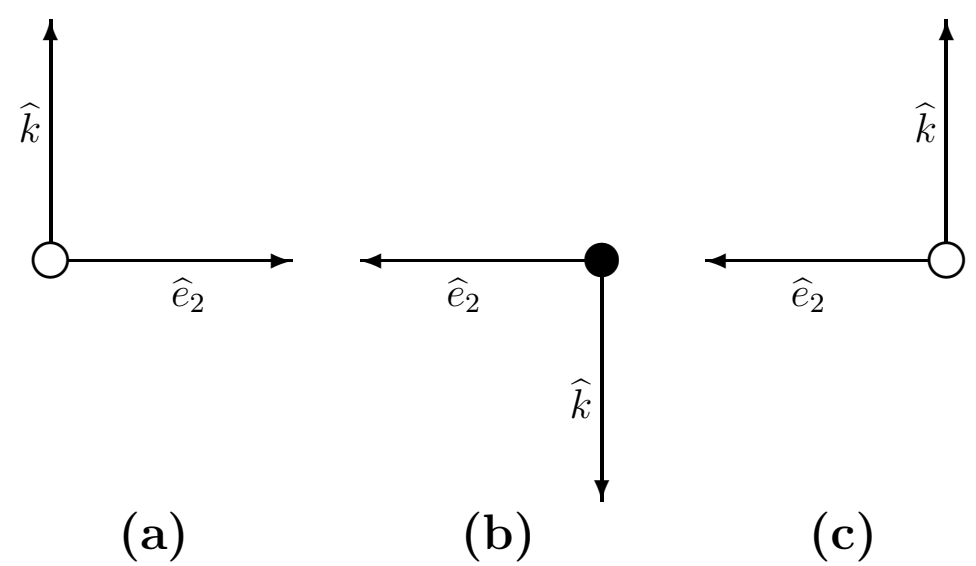

Figure $\mathrm{O}$

Figure 2: The effect of a Parity transformation on the wave vector $\mathbf{k}$ and the transverse polarization vectors. The empty circles in diagrams (a) and (c) indicate that the direction of $\hat{e}_{1}$ is towards the reader, whereas the filled circle in diagram (b) indicates the direction to be into the paper.

this corresponds to absorption by (or emission from) the medium. Even in the case of $\zeta=0$, absorption of the two transverse modes can arise if $\epsilon$ or $\mu$ is complex. But the absorption has to be equal for the two modes. For $\zeta \neq 0$, that is not the case.

For the sake of simplicity, we will stick to an unabsorbing medium. This means that $\omega$ is real for all wave vectors k. From Eq. (5.7), this implies that $\epsilon, \mu$, are real, $\zeta$ is purely imaginary and $|\zeta|<k c|\mu| / \omega$. As already remarked, in this simple case the effect of a non-zero value of $\zeta$ is to produce a difference in speed between the two circularly polarized degrees of freedom.

\section{$6 \quad$ Physical implications of $\zeta$}

We now discuss the conclusions of the last section in a more intuitive way on the basis of the discrete operations P, C and T. Again, we concentrate on the transverse modes only, and draw the polarization vectors $\hat{e}_{1}, \hat{e}_{2}$ along with the unit wave-vector $\hat{k}$ in Fig. 2 a.

Now imagine the effect of parity on the vectors in Fig. 2a. Surely $\hat{k}$ reverses direction, since it is a polar vector denoting the direction of wave propagation. To see the effect of parity on $\hat{e}_{1}$ and $\hat{e}_{2}$, we first need a precise definition of them. This depends on the way we choose the scalar and vector potentials to express the electric and magnetic fields. For the sake of convenience, we take the "radiation gauge" in which the scalar potential vanishes and

$$
\mathbf{E}=i \omega \mathbf{A}, \quad \mathbf{B}=i \mathbf{k} \times \mathbf{A} .
$$

In this gauge, we can express the vector potential as

$$
\mathbf{A}=\sum_{\lambda} a_{\lambda} \hat{e}_{\mathbf{k} \lambda}
$$

where the quantities $a_{\lambda}$ are numerical coefficients in classical electromagnetism. Now, under parity, E reverses sign, so that from Eq. (6.1), we see that $\mathbf{A}$ must do the same. From Eq. (6.2), we then obtain that the vectors $\hat{e}_{\mathbf{k} \lambda}$ must also change sign. All of these facts have been summarized in Fig. $2 \mathrm{~b}$. 
However, since we are dealing with isotropic systems, the absolute directions of the vectors do not matter, only their relative orientations do. Thus, rotating Fig. $2 \mathrm{~b}$ around the $\hat{e}_{2}$-axis by $180^{\circ}$, we obtain Fig. 20. Comparing this with Fig. 2a, we see that effectively, for the same $\hat{k}$, the effect of the parity transformation has been to reverse the sign of $\hat{e}_{2}$.

An alternative way to discuss the polarization of electromagnetic waves is to use, instead of the linear polarization vectors $\hat{e}_{1}$ and $\hat{e}_{2}$, the right and left circular polarization vectors defined by

$$
\hat{e}_{ \pm} \equiv \frac{1}{\sqrt{2}}\left(\hat{e}_{1} \pm i \hat{e}_{2}\right) \text {. }
$$

Since $\hat{e}_{2}=\hat{k} \times \hat{e}_{1}$, these are of the form $\hat{e}_{1} \pm i \hat{k} \times \hat{e}_{1}$, as mentioned after Eq. (5.8). In this language, thus, the effect of parity is easily understood. Since effectively $\hat{e}_{2} \longrightarrow-\hat{e}_{2}$, as shown earlier, we conclude that under parity

$$
\hat{e}_{+} \longleftrightarrow \hat{e}_{-}
$$

Thus, if parity is a good symmetry, there should be no difference in the physical properties of a rightcircular and a left-circular polarization state of an electromagnetic wave. Such is the case, for example, in the vacuum, since Eqs. 2.5) and (2.6) are invariant under parity.

In matter, as we pointed out, this is not the case. Eq. (3.3) and its corollary in Eq. (4.6) break parity because of the $\zeta$-term. Thus, in a medium, the right- and the left- circularly polarized electromagnetic waves can have different physical properties. For example, they travel with different speeds through matter, have different indices of refraction and coefficients of absorption.

It is easy to see that this difference manifests itself as optical activity. Consider a plane polarized wave incident on a medium at $x=0$, moving in the positive $x$-direction. Suppose the direction of polarization of the incident wave is along $\varphi=0$, where $\varphi$ denotes the azimuthal angle in the plane perpendicular to the direction of propagation. As is well-known [18], this plane polarized wave can be treated as the superposition of a right-circularly polarized wave with phase angle $\varphi_{+}(0)=\omega t$ and a left-circular polarized wave of the same amplitude with phase angle $\varphi_{-}(0)=-\omega t$. The zero in the paretheses here denote the fact that these phases are given for the interface where $x=0$.

Now the wave propagates inside the medium, where $\zeta \neq 0$, and consequently, the same value of $\omega$ corresponds to different values of the wave number $k$ for the two circularly polarized components, as we argued before. Let us call these values of $k$ by $k_{+}$and $k_{-}$. Then, after traversing a distance $x$ within the medium, the phase angle for the right- and left- circularly polarized components become

$$
\varphi_{+}(x)=\omega t-k_{+} x, \quad \varphi_{-}(x)=-\omega t+k_{-} x,
$$

so that the resultant direction is given by

$$
\varphi(x)=\frac{1}{2}\left[\varphi_{+}(x)+\varphi_{-}(x)\right]=\frac{1}{2}\left(k_{-}-k_{+}\right) x .
$$

This shows that the direction of polarization of the wave changes with $x$ within the medium, which is precisely the phenomenon of optical activity.

A similar treatment can be applied to the phenomenon that occurs in the optical-rotation experiments that have been performed to test the parity-violating weak force between the electrons and nucleons, which coexists with the electromagnetic force [19]. In these experiments, a beam of lineraly polarized light traverses a cell containing atomic vapor of bismuth, and the direction of polarization of the wave changes as the beam traverses the cell. From a macroscopic point of view, the effect can be understood and described as discussed above.

The last two equations, together with our earlier analysis leading to Eq. (5.7), bring out clearly the fact that the difference between $k_{-}$and $k_{+}$is introduced because $\zeta \neq 0$ [20]. In view of this, the quantity $\zeta$ may be called the activity constant of a medium. 


\section{Conclusions and summary}

We have explained why, in order to describe electromagnetic phenomena in an isotropic medium, one needs in general three parameters, or electromagnetic constants. Two of them are introduced and studied in detail in every textbook on Electromagnetic Theory, and are called the dielectric constant and the magnetic permeability of the medium in question. The third one, which we denote by the symbol $\zeta$ in this paper, is not as widely discussed or not introduced at all in physics textbooks. We showed that $\zeta$ has some features which are very different from the other two. For example, a medium can have a non-zero value of $\zeta$ only if there are asymmetries of parity, CP etc. These asymmetries may come either from a violation of those symmetries in fundamental interactions between particles, or from the constituents of the medium. Since it is known that particle interactions violate parity and $\mathrm{CP}$, the value of $\zeta$ is in principle non-zero for all normal media. Morever, if the constituents of the medium are parity-asymmetric objects like helical molecules, the effect can be large.

Physically, the presence of the $\zeta$ term introduces differences between the characteristics of the right- and left-circularly polarized electromagnetic waves. These differences manifest themselves in various physical phenomena, e.g. optical activity and atomic parity violation experiments. Our approach in the present paper provides a general framework to study and interpret these phenomena from a unified point of view.

\section{Appendix}

\section{A Time reversal of the Maxwell equations in Classical and Quantum mechanics}

As we have discussed in the text, a non-zero value of $\zeta$ has deep implications regarding the discrete spacetime symmetries of the system. The transformation rule that we have used for Time-Reversal is apropriate in the context of Classical Physics which, for clarity, we have adopted in the text. In the realm of Quantum Mechanics that definition of Time-Reversal cannot be used. Therefore, it is important to discuss the appropriate transformation rule in Quantum Mechanics, since in principle $\zeta$ has to be calculated in that context. In this appendix, we discuss the different definitions of the time-reversal transformation and why they arise.

Let us use as starting point Eq. (4.6) which gives the external current density in terms of the electric and magnetic fields. For the sake of convenience, we define the following quantities:

$$
\begin{aligned}
L_{E}^{a b}(\mathbf{k}, \omega) & =i \omega \epsilon \delta^{a b} \\
L_{B}^{a b}(\mathbf{k}, \omega) & =\frac{c}{\mu} i \varepsilon^{a c b} k^{c}+i \omega \zeta \delta^{a b},
\end{aligned}
$$

where the superscripts $a, b$ denote Cartesian components, $\delta^{a b}$ denotes the Kronecker delta symbol and $\varepsilon^{a c b}$ is the completely antisymmetric tensor with $\varepsilon^{123}=+1$. With these, we can rewrite Eq. (4.6) as

$$
\mathbf{j}_{\mathrm{ext}}^{a}=\sum_{b} L_{E}^{a b} \mathbf{E}^{b}+L_{B}^{a b} \mathbf{B}^{b} .
$$

Since $\mathbf{j}_{\text {ext }}$ and $\mathbf{B}$ change sign under time reversal whereas $\mathbf{E}$ does not, we can say that the Time-Reversal invariance of the Maxwell equations is equivalent to the statement that $L_{E}$ and $L_{B}$ have the following properties:

$$
\begin{aligned}
& L_{E}(\mathbf{k},-\omega)=-L_{E}(\mathbf{k}, \omega) \\
& L_{B}(\mathbf{k},-\omega)=L_{B}(\mathbf{k}, \omega) .
\end{aligned}
$$


These in turn imply the relations on $\epsilon, \mu$ and $\zeta$ that have been obtained in the text. This definition of the Time-reversal operation can be called the classical definition. If Eq. (A.3) holds, then the Maxwell equations are invariant under the substitutions

$$
\begin{aligned}
& \mathbf{E}(\mathbf{k}, \omega) \stackrel{T}{\longrightarrow} \mathbf{E}^{\prime}\left(\mathbf{k}^{\prime}, \omega^{\prime}\right) \equiv \mathbf{E}(\mathbf{k}, \omega), \\
& \mathbf{B}(\mathbf{k}, \omega) \stackrel{T}{\longrightarrow} \mathbf{B}^{\prime}\left(\mathbf{k}^{\prime}, \omega^{\prime}\right) \equiv-\mathbf{B}(\mathbf{k}, \omega) \text {, } \\
& \mathbf{j}(\mathbf{k}, \omega) \stackrel{T}{\longrightarrow} \mathbf{j}^{\prime}\left(\mathbf{k}^{\prime}, \omega^{\prime}\right) \equiv-\mathbf{j}(\mathbf{k}, \omega),
\end{aligned}
$$

where

$$
(\mathbf{k}, \omega) \stackrel{T}{\longrightarrow}\left(\mathbf{k}^{\prime}, \omega^{\prime}\right) \equiv(\mathbf{k},-\omega) .
$$

Eq. A.4 is, of course, equivalent to the identification of the value of $\eta_{T}$ for the various quantities given in Table 2.

In Quantum Mechanics, however, these transformation rules cannot be used. The reason is that, while they leave the Maxwell equations invariant, these rules do not leave invariant the quantum mechanical equations of motion for the charges, namely the Schrodinger equation. A simple way to understand this is to recall that, in Quantum Mechanics, one identifies $\omega=\mathcal{E} / \hbar$ and $\mathbf{k}=\mathbf{p} / \hbar$, where $\mathcal{E}$ and $\mathbf{p}$ denote the energy and momentum of the particle and $\hbar$ is Planck's constant divided by $2 \pi$. Now, from the non-relativistic definitions of momentum and kinetic energy of a point particle [viz., $\mathbf{p}=m \frac{d \mathbf{r}}{d t}$ and $\mathcal{E}_{\text {kin }}=\frac{1}{2} m\left(\frac{d \mathbf{r}}{d t}\right)^{2}$ ], one expects that $\mathbf{p}$ changes sign under time-reversal whereas $\mathcal{E}$ does not. Thus, instead of Eq. (A.5), the transformation is defined by

$$
(\mathbf{k}, \omega) \stackrel{T}{\longrightarrow}\left(\mathbf{k}^{\prime}, \omega^{\prime}\right) \equiv(-\mathbf{k}, \omega) .
$$

This is how the transformation must be defined in order to leave the Schrodinger equation for a free particle invariant. The interesting point is that this transformation is a symmetry operation of the Maxwell equations also, provided that $L_{E}$ and $L_{B}$ satisfy, instead of Eq. (A.3), the following:

$$
\begin{aligned}
& L_{E}^{*}(-\mathbf{k}, \omega)=-L_{E}(\mathbf{k}, \omega), \\
& L_{B}^{*}(-\mathbf{k}, \omega)=L_{B}(\mathbf{k}, \omega) .
\end{aligned}
$$

If these relations hold, then the Maxwell equation is invariant under the substitutions:

$$
\begin{aligned}
& \mathbf{E}(\mathbf{k}, \omega) \stackrel{T}{\longrightarrow} \mathbf{E}^{\prime}\left(\mathbf{k}^{\prime}, \omega^{\prime}\right) \equiv \mathbf{E}^{*}(\mathbf{k}, \omega) \\
& \mathbf{B}(\mathbf{k}, \omega) \stackrel{T}{\longrightarrow} \mathbf{B}^{\prime}\left(\mathbf{k}^{\prime}, \omega^{\prime}\right) \equiv-\mathbf{B}^{*}(\mathbf{k}, \omega) \\
& \mathbf{j}(\mathbf{k}, \omega) \stackrel{T}{\longrightarrow} \mathbf{j}^{\prime}\left(\mathbf{k}^{\prime}, \omega^{\prime}\right) \equiv-\mathbf{j}^{*}(\mathbf{k}, \omega) \\
& i \stackrel{T}{\longrightarrow} \quad i^{\prime} \quad \equiv i^{*}=-i .
\end{aligned}
$$

We will refer to these transformation rules as the Quantum Mechanical ones. Since the transformation rules are different from the ones discused before, the consequences of time reversal symmetry on $\epsilon, \mu$ and $\zeta$ change as well.

The last comment is meant to emphasize that Eqs. (A.4) and (A.8) are two different transformation rules, and the symmetries that they imply are also different. However, they are equivalent if, but only if, $L_{E}$ and $L_{B}$ do not have an absorptive part. In that case, those quantities depend on $i$ only through the combinations $i \mathbf{k}$ and $i \omega$, and therefore taking the complex conjugate of them is the same as changing the sign of $\omega$ and $\mathbf{k}$ in their argument. It is then immediately seen that Eqs. (A.3) and (A.7) are really the same. However, if $L_{E}$ and $L_{B}$ have an absorptive part, then the two transformation laws are different. 
The classical transformation is a symmetry operation only if Eq. A.3 holds. That equation implies the relations which have been derived in Eq. (4.13), which in turn imply that $\epsilon, \mu$ are real while $\zeta$ is purely imaginary; i.e., neither have an absorptive part. The classical transformation law is not a symmetry operation if any of those quantities has an absorptive part. However, the quantum mechanical transformation law is a symmetry operation even when they have an absorptive part; what is needed is that Eq. (A.7) be satisfied, and that is always true because $\epsilon, \mu$ and $\zeta$ satisfy the relation (4.8). As already discused, in classical physics this relation is a consequence of the fact that the fields and the current are real quantities, while in Quantum Mechanics it is just the requirement of Hermiticity of the current and the fields.

We summarize the situation as follows. In a non-absorbing medium, the classical and quantum definitions of the Time-Reversal operation coincide. However, the classical Time-Reversal symmetry is broken if $\epsilon, \mu$ or $\zeta$ have an absorptive part. Therefore, in the realm of Classical Physics, it is impossible to get an absorptive part of those quantities except if the theory breaks the (classical) Time-Reversal symmetry. The Schrodinger equation breaks that symmetry; quantum effects break that transformtion. But the equations of Quantum Mechanics (and of course Maxwell equations) are invariant under a more general transformation, which continues to hold even when $\epsilon, \mu$ and $\zeta$ have an absorptive part. The existence of the $\zeta$ term implies that $\mathrm{P}$ is broken but not $\mathrm{T}$ (with the quantum mechanical definition of $\mathrm{T}$ ) nor $\mathrm{C}$. Thus, $\zeta$ breaks $\mathrm{P}, \mathrm{CP}$ and $\mathrm{CPT}$.

\section{References}

[1] See any text in electrodynamics, e.g., E. M. Purcell, Electricity and Magnetism, (Berkeley Physics Course, vol. 2; McGraw-Hill, New York 1989), Chaps. 10-11; D. Halliday and R. Resnick, Fundamentals of Physics (John Wiley \& Sons, New York 1986) Chap. 27.

[2] See, for example, E. U. Condon, Theories of optical rotatory power, Rev. Mod. Phys. 9, 432-457 (1937); P. Drude, The theory of optics, (Dover, 1959); E. J. Post, Fundamental Structure of Electromagnetics, (North-Holland, 1962); E. Charney, The molecular basis of optical activity (Wiley, New York 1979); A. Lakhtakia, V. K. Varadan and V. V. Varadan, Time-Harmonic Electromagnetic fields in Chiral Media, (Springer, 1989); J. A. Kong, Electromagnetic Wave Theory, (John Wiley \& Sons, 1986).

[3] There are exceptions, e.g., the book by L. D. Landau and E. M. Lifshitz, Electrodynamics of Continuos Media, Course of Theoretical Physics, Volume 8, (Pergamon Press, 1960) pp 337.

[4] J. F. Nieves and P. B. Pal, P- and CP-odd terms in the photon self-energy within a medium, Phys. Rev. D 39, 652-659 (1989).

[5] J. F. Nieves and P. B. Pal, Propagation of gauge fields within a medium, Phys. Rev. D 40, 1350-1353 (1989).

[6] More commonly, one expresses the dimension of charge can be expressed in terms of the fundamental dimensions of length, time and mass through the Coulomb force law. However, since the mass dimension is not directly necessary for our purpose, we use length, time and charge as the fundamental units in this paper.

[7] In general, what we need to do is to look for solutions which are expressed as a superposition of plane waves, with $\mathbf{k}$ and $\omega$ not being necessarily real. Such waves are called inhomogeneous plane waves, and they form a general basis for the treatment of boundary-value problems for waves. Those waves in general exhibit exponential growth or decay in time, and also in space along some directions, some 
simple examples being the phenomena of total internal reflection and refraction in conducting media. For our present concerns, we are considering a spatially infinite medium, in which case $\mathbf{k}$ must be taken to be real, but $\omega$ must in general be allowed to be complex. Therefore, in Eq. (2.3), $\mathbf{k}$ is real but $\omega$ can be complex, and the integral over $\omega$ must be understood accordingly. For further discussion about these points see, e.g., J. D. Jackson, Classical Electrodynamics, (John Wiley \& Sons, New York 1975) Chap. 7.

[8] In the most general case $\epsilon$ and $\mu^{-1}$ are rank-2 tensors, and the terms denoted by 1 in Eq. (2.10) stand for the unit tensor.

[9] J. C. Monzon, Radiation and scattering in homogenoeous general biisotropic regions, IEEE Trans. Ant. Prop. 38, 227-235 (1990).

[10] C. M. Krowne, Electromagnetic theorems for complex anisotropic media, IEEE Trans. Antennas Propag. 32, 1224-1230 (1984).

[11] J. A. Kong, Theorems of bianisotripic media, Proc. IEEE 60, 1036-1046 (1972).

[12] B. D. H. Tellegen, The gyrator, a new network element, Phillips Res. Rept. 3, 81-101 (1948).

[13] See, e.g., R. A. Satten, Time Reversal Symmetry and Electromagnetic Polarization Fields, J. Chem. Phys. 28, 742-743 (1958).

[14] We are discussing the Time-Reversal operation here in classical terms, and we will keep that spirit in the rest of the paper. In the appendix we discuss the definition that must be adopted in the realm of Quantum Mechanics and also show that in a non-absorbing medium the two definitions coincide.

[15] Protons might be present in order to attain a neutral medium. However, their presence does not affect our conclusion.

[16] In Eq. (5.3) it cannot be assumed that $\hat{k} \times \mathbf{E}$ and $\mathbf{E}$ are linearly independent, and therefore we cannot conclude that the coefficient of the last term must be zero. For a complex vector $\mathbf{E}, \hat{k} \times \mathbf{E}$ and $\mathbf{E}$ can be linearly dependent.

[17] In fact, it is easy to prove (see, e.g., Condon, Ref. [2]) that the left- and right- circular polarized waves are the only ones which can travel through the medium with unchanged state of polarization. For this, write Eqs. (5.3) and (5.5) in a matrix form:

$$
\left(\begin{array}{cc}
\omega^{2}-\frac{c^{2}}{\epsilon \mu} k^{2} & \frac{c \zeta}{\epsilon} \omega k \\
-\frac{c \zeta}{\epsilon} \omega k & \omega^{2}-\frac{c^{2}}{\epsilon \mu} k^{2}
\end{array}\right)\left(\begin{array}{c}
\mathbf{E} \\
\hat{k} \times \mathbf{E}
\end{array}\right)=0 .
$$

This is an eigenvalue problem whose characteristic equation yields the dispersion relations of Eq. (5.7), with the eigenvectors determined as the solutions of Eq. (5.8).

[18] Various textbooks on Optics discuss this. See, e.g., B. Rossi, Optics, (Addison-Wesley, Reading 1957) Chap. 6.

[19] See, e.g., E. D. Commins and P. H. Bucksbaum, Weak Interactions of leptons and quarks, (Cambridge University Press 1983) pp 349.

[20] Explicit solutions of Eq. (5.7), which clearly illustrate how the dispersion relations for right- and leftcircularly polarized waves differ, can be readily obtained for the cases in which $\epsilon$ and $\mu$ are constants and $\zeta$ is proportional to either $\omega, \omega^{-1}, \omega^{3}$ or $\omega^{-3}$. On the other hand, perturbative solutions can be obtained in the case that $\zeta$ is small. 